\title{
Evolution de l'ultrastructure du pancréas chez le foetus de rat
}

\author{
H. Perrier \\ avec la collaboration technique de C. Brusat \\ Laboratoire de Physiologie Animale et Centre de Biologie et Biochimie du Développement, Faculté des Sciences Reims, \\ France
}

Reçu le 4 mai 1970

\begin{abstract}
Development of the ultrastructure of the foetal rat pancreas

Summary. The development of the endocrine and exocrine cells of Wistar rat pancreas was studied from day 16 through day 21 of gestation by ultrastructural observations. - A and B endocrine cells appeared immature on day 16 through day 18, being rich in free ribosomes and having relatively few and small secretory granules on day 16 and 17. These cells were dispersed among morphologically undifferenciated cells. On day 18, however, cells of this type were grouped into small islets. The maturation of the endocrine tissue starting on day 19 could be identified by the organization of the adult type of the islets (peripheral A cells) and by the development of the cellular ultrastructure, both being evident on day 20. In particular, the maturation of the B cells was indicated by the growing number of secretory granules, which were obviously more voluminous than in the earlier stages of development, and by the growth of the ergastoplasmic and Golgian structures. The maturation of the A cells was manifested by an increase in size and number of the secretory granules. The morphologically differentiated exocrine cells were numerous on day 17 . The zymogen granules were numerous from day 18 on. At the same time the size of the granules increased and the ergastoplasmic structures were clearly developed. Later, only minor ultrastructural modifications could be observed.
\end{abstract}

Résumé. L'observation ultrastructurale du pancréas des foetus de rats Wistar, âgés de $16,17,18,19,20$ et 21 jours a permis d'étudier l'évolution du pancréas endocrine et exocrine au cours de la fin de la gestation. - Des cellules endocrines A et B présentant, par leur richesse en ribosomes libres, leurs granules sécrétoires relativement peu nombrenx et de petite taille, un aspect peu mature sont à 16 et 17 jours dispersées parmi les cellules morphologiquement indifférenciées et à 18 jours groupées en petits îlots. La maturation du tissu endocrine amorcée à 19 jours se manifeste à 20 jours par l'organisation de type adulte des îlots (cellules A périphériques) et par l'évolution ultrastructurale des cellules. La matura- tion des cellules B se traduit par un enrichissement en granules sécrétoires, qui sont nettement plus volumineux qu'aux stades précédents, et un développement des structures ergastoplasmiques et golgiennes. Dans les cellules A la maturation se manifeste par une augmentation de la taille et du nombre des granules sécrétoires. Les cellules exocrines morphologiquement différenciées deviennent nombreuses à 17 jours; mais ne sont riches en grains de zymogène qu'à partir de 18 jours. Cet enrichissement s'accompagne d'un accroissement de la taille des grains et d'un développement des structures ergastoplasmiques. Aux stades suivants l'évolution est discrète. kreas

Entwicklung der Ultrastruktur des foetalen Rattenpan-

Zusammenfassung. Die Entwicklung der exokrinen und endokrinen Zellen des Pankreas von 16, 17, 18, 19, 20 und 21 Tage alten Föten wurde elektronenmikroskopisch untersucht. - Bis zum 18. Tag haben die endokrinen Zellen (A und B) noch ein unreifes Aussehen (verhältnismäßig wenig kleine Sekretionskörnchen und zahlreiche freie Ribosome). Am 16. und 17. Tag liegen sie spärlich zwischen morphologisch undifferenzierten Zellen, vom 18. Tage an bilden sie kleine Inseln. Das Reifen der endokrinen Zellen beginnt am 19. Tag und wird am 20. Tag mit dem Erreichen der beim Erwachsenen sichtbaren Inselorganisation, d.h. mit ihrer peripheren A Zellenlage besonders deutlich. Die Ultrastruktur der endokrinen Zellen verändert sich. Während die B Zellen eine Vermehrung der jetzt deutlich größeren Sekretionskörnchen und eine Ausdehnung des Ergastoplasmas und des Golgi Apparates vorweisen, zeigen die A Zellen eine Zunahme der Zahl und Größe der Sekretionskörnchen. - Die morphologisch vollentwickelten exokrinen Zellen werden am 17. Tag zahlreich. Am 18. Tag weisen sie eine Erhöhung der Zahl und Größe der Zymogenkörnchen auf und eine größere Ausdehnung des Ergastoplasmas. Nach dieser Zeit treten nur geringe strukturelle Veränderungen auf.

Key-words: Endocrine pancreas, exocrine pancreas, foetal rat, ultrastructure.
De nombreux types cellulaires ont été décrits dans le pancréas endocrine de rat et de souris adultes $[1,12$, $15,19]$. Si certaines catégories sont fortement contestées deux sont toujours reconnues: cellules de type A et cellules de type $\mathrm{B}$. Les cellules $\mathrm{A}$ sont préférentiellement disposées à la périphérie des îlots, les cellules $\mathrm{B}$ étant plus centrales. Au microscope électronique les cellules B apparaissent plus denses que les cellules A car leur cytoplasme est plus riche en ergastoplasme. Leur appareil de Golgi est plus développé. Leur matériel sécrétoire se présente sous forme de vésicules de taille variable renfermant un grain dense à tendance cristalloïde. Par contre le matériel sécrétoire des cellules A est formé de granules sphériques électroniquement denses, séparés de la membrane limitante par un discret espace clair. Du point de vue physiologique les cellules $\mathrm{B}$ sont reconnues depuis longtemps comme productrices d'insuline et on sait maintenant qu'au moins une partie des cellules $\mathrm{A}$ (cellules $\mathrm{A}_{2}$ ) sécrète du glucagon $[10,11,22]$.

Des expériences montrent que le pancréas du foetus de rat est capable de synthétiser et d'excréter de l'insuline $[3,7,9,16,17,26]$ et certaines données physiologiques appuient l'hypothèse d'une sécrétion 
de glucagon en fin de gestation $[6,13]$. Depuis les travaux de Hard [8] on admettait généralement que chez le foetus de rat et de souris les cellules $B$ se différencient à partir du 18ème jour de gestation; divers auteurs ont cependant observé une différenciation plus précoce, vers le 13ème jour $[19,23]$ et même dès le 11ème jour [27]. De même les cellules A n'ont pas été observées avant la naissance par Ferreira [4], Gonet [5], Hard [8], Hellman [12], Munger [19] mais des travaux récents $[20,21$,$] ont montré la$ présence de ces cellules chez le foetus.

Nous avons fait une étude ultrastructurale systématique du pancréas foetal de rat dans le but de plus $10 \mathrm{~h}$; par simplification nous dirons $\mathbf{n}$ jours (c'est-à-dire le $(n+1)$ ème jour de gestation). Les pancréas sont prélevés chez les foetus de 16, 17, 18, $19,20,21$ jours et chez les jeunes de 8 jours.

Les échantillons sont fixés 5 à $10 \mathrm{mn}$ à la glutaraldéhyde, post-fixés 1 heure à l'acide osmique et inclus dans l'araldite. L'observation de coupes semi-fines au microscope à contraste de phase permet de choisir les régions les plus riches en cellules endocrines qui apparaissent plus pâles, dépourvues de grains de zymogène. Les coupes ultra-fines sont contrastées à l'acétate d'uranyle-citrate de plomb et étudiées au microscope électronique Hitachi Type H.S.8.

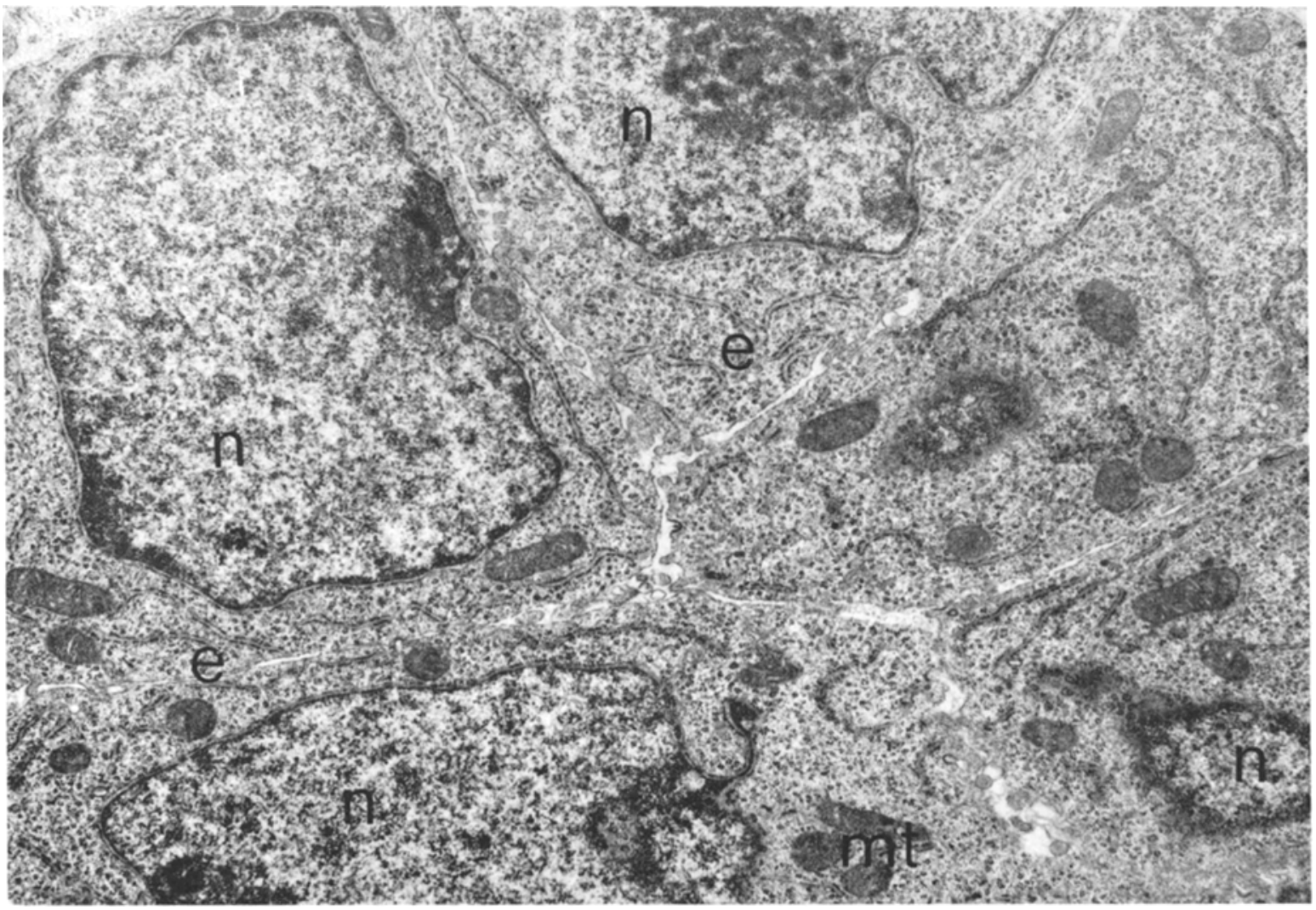

Fig. 1. Foetus de 16 jours. Cellules morphologiquement indifférenciées, eiternes ergastoplasmiques longues et flexueuses $(\epsilon)$, très nombreux ribosomes libres. $n$ : noyau. $m t$ : : mitochondries $(\times 11600)$

préciser l'évolution des cellules A et B. Simultanément quelques observations sur le tissu exocrine sont rapportées.

\section{Technique}

Les rattes (souche Wistar C.F. du C.N.R.S.) sont mises en présence de mâles pendant la nuit. La découverte de spermatozoïdes dans le frottis vaginal le lendemain matin permet de fixer le début de la gestation. On supposera arbitrairement que la copulation a eu lieu à minuit ce qui marque l'origine du temps. Les prélèvements sont toujours effectués vers $10 \mathrm{~h}$ du matin et les foetus sont donc âgés de $\mathrm{n}$ jours
Résultats

$A 16$ jours, l'observation au microscope à contraste de phase ne permet pas de distinguer pancréas endocrine et pancréas exocrine.

Au microscope électronique, la presque totalité des cellules présente un aspect morphologiquement indifférencié. Le cytoplasme est extrêmement riche en ribosomes libres, souvent groupés en polysomes, et renferme quelques citernes ergastoplasmiques longues et flexueuses, à contenu dense. L'appareil de Golgi est peu développé. Les mitochondries sont peu nombreuses mais relativement volumineuses et riches en crêtes internes (Fig. 1). Les figures de mitose sont fréquentes. 
Nous avons pu observer, déjà à ce stade, de rares cellules contenant des granules sécrétoires denses et arrondis, séparés de leur membrane limitante par un discret espace clair. Ce type de granules est caractéristique des cellules A. Ces cellules présentent par ailleurs la même richesse en ribosomes que les cellules morphologiquement non différenciées voisines; mais leur appareil de Golgi est plus développé. Elles contiennent trouver car, comme à 16 jours, elles sont dispersées parmi des cellules morphologiquement indifférenciées. On observe des cellules A présentant le même aspect qu'au stade précédent. Des cellules B sont également identifiables par leurs granules sécrétoires à tendance cristalloïde situés dans des vésicules claires; la totalité des granules sécrétoires de type B ne présente pas toutefois cet aspect caractéristique et certains d'entre

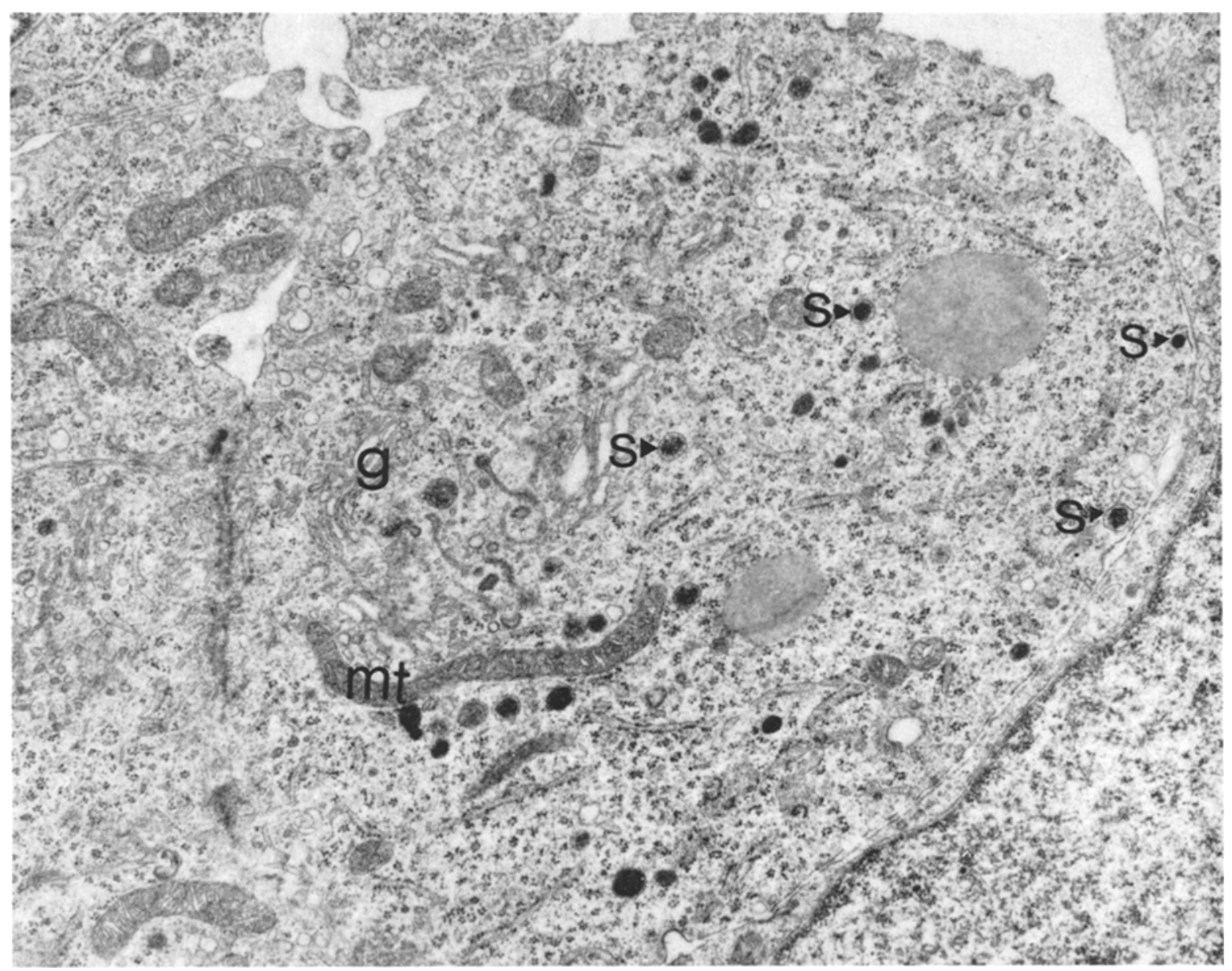

Fig. 2. Foetus de 16 jours. Cellules A, granules sécrétoires $(s)$ peu nombreux, grande richesse en ribosomes libres. $g$ : golgi. mt.: mitochondries $(\times 17400)$

des mitochondries allongées et des inclusions lipidiques relativement volumineuses (Fig. 2). Nous n'avons pas observé de cellules $\mathrm{B}$, ce qui n'exclut pas leur présence à ce stade. Il faut remarquer en effet qu'à 16 jours les cellules endocrines (A ou $B$ ) sont isolées au milieu de cellules morphologiquement indifférenciées; la probabilité de les repérer est donc beaucoup plus faible que lorsqu'elles sont regroupées en îlots que l'on peut reconnaître au microscope à contraste de phase et sélectionner pour l'observation en microscopie électronique.

A 17 jours, les cellules endocrines en voie de différenciation morphologique sont encore difficiles à eux ressemblent aux granules des cellules A; ces cellules $B$ sont plus riches en structures ergastoplasmiques que les cellules $A$. Le nombre des granules de sécrétion (de type A ou B) varie assez nettement d'une cellule à l'autre (Figs. 3 et 4 ).

Le phénomène le plus marquant à 17 jours est le début de la différenciation morphologique d'un grand nombre de cellules exocrines, marqué par l'apparition de volumineux grains denses (les plus gros mesurant $680 \mathrm{~nm}$ ) qui tendent à s'accumuler au pôle apical, en bordure des canalicules. Ces grains de zymogène sont généralement peu nombreux, et, comme dans le cas des cellules endocrines, la richesse en matériel sécrétoire 


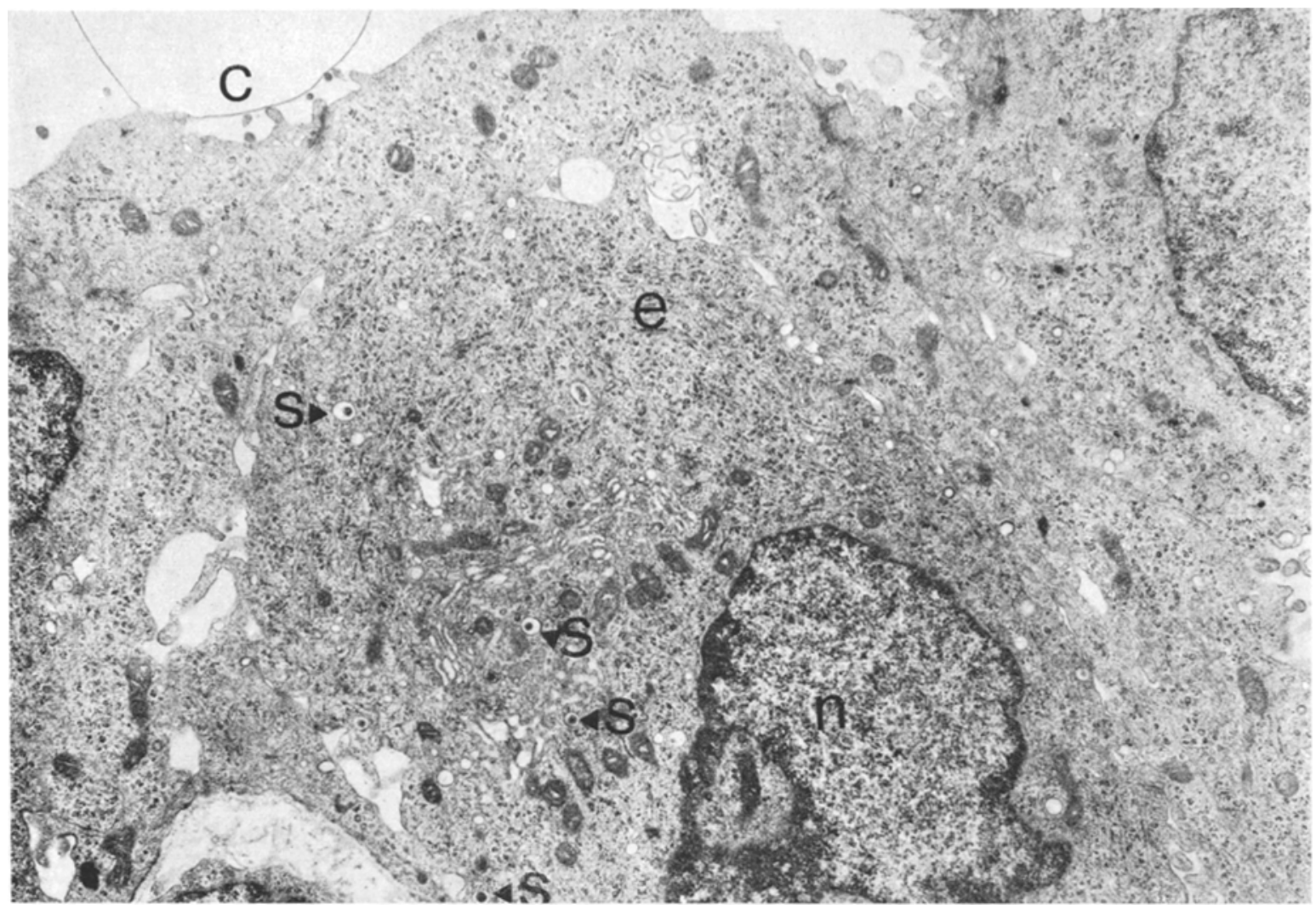

Fig. 3. Foetus de 17 jours. Cellules B dans la paroi d'un canalicule (c). Quelques rares granules sécrétoires (s), ergastoplasme (e) bien développé. $n$ : noyau. $(\times 9000)$

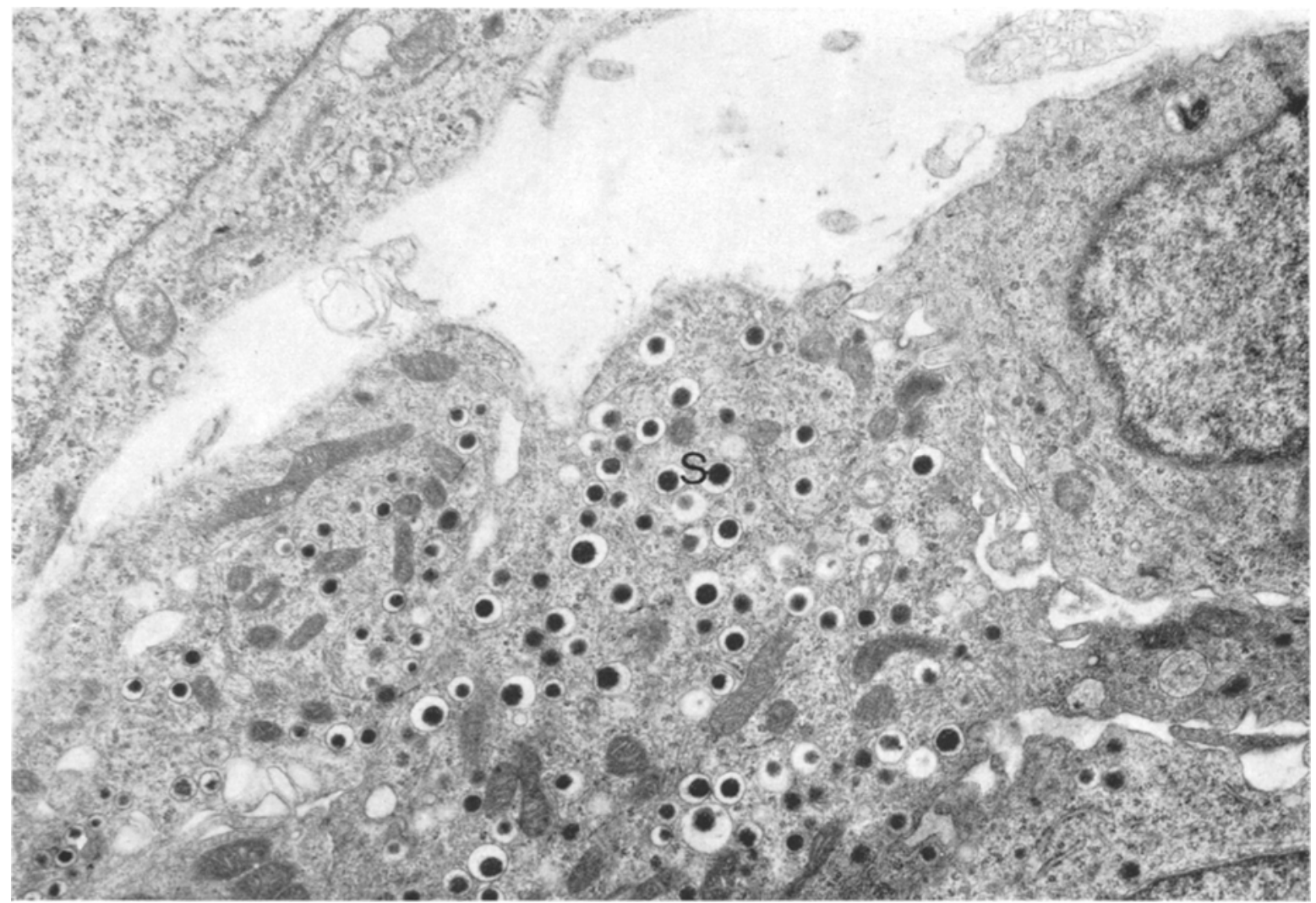

Fig. 4. Foetus de 17 jours. Cellules $\mathrm{B}$ assez riches en granules $(s)(\times 14580)$ 


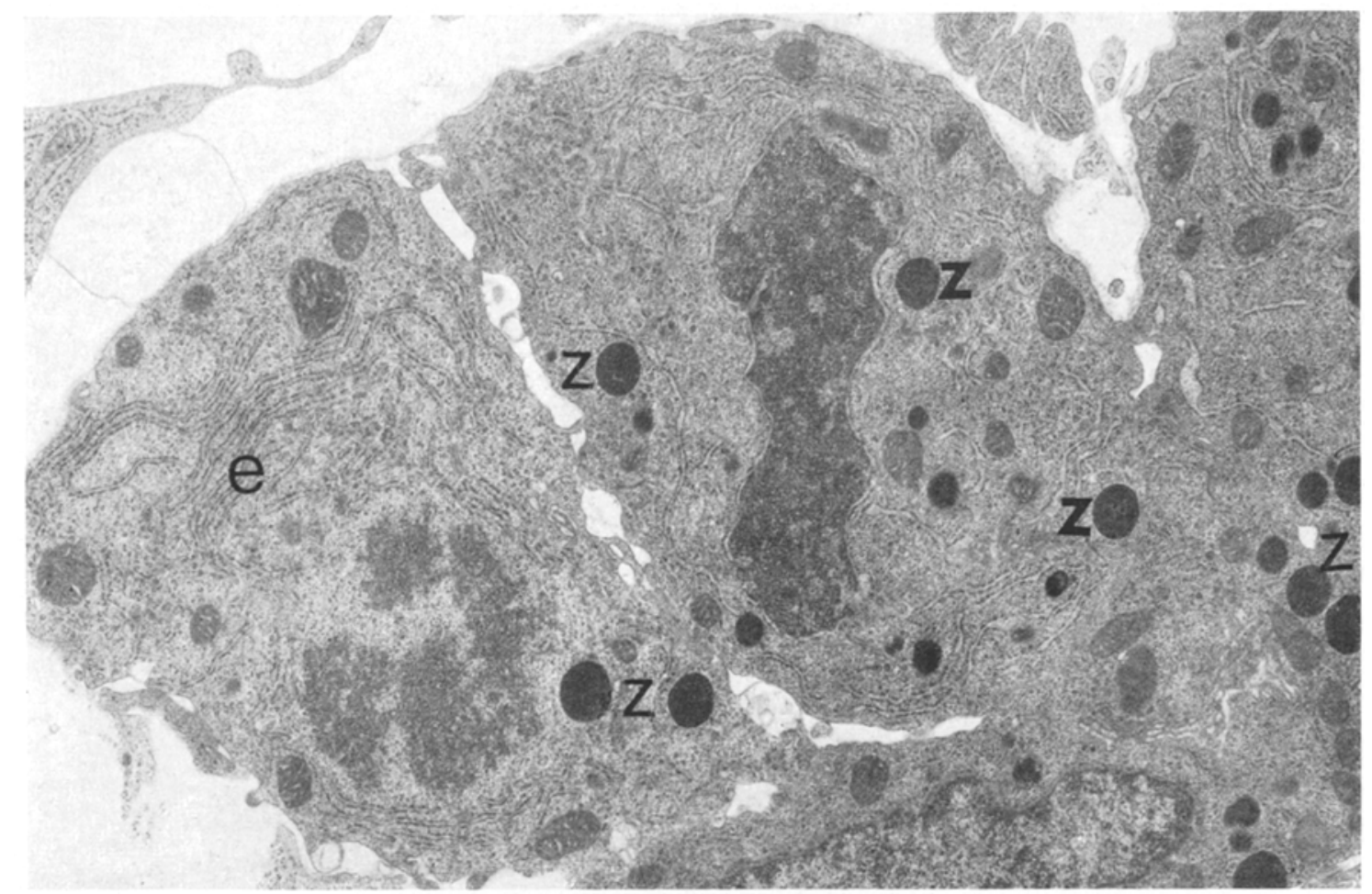

Fig. 5. Foetus de 17 jours. Cellules exocrines. Grains de zymogène $(z)$ assez volumineux, ergastoplasme $(e)$ important, fin de mitose $(\times 8940)$

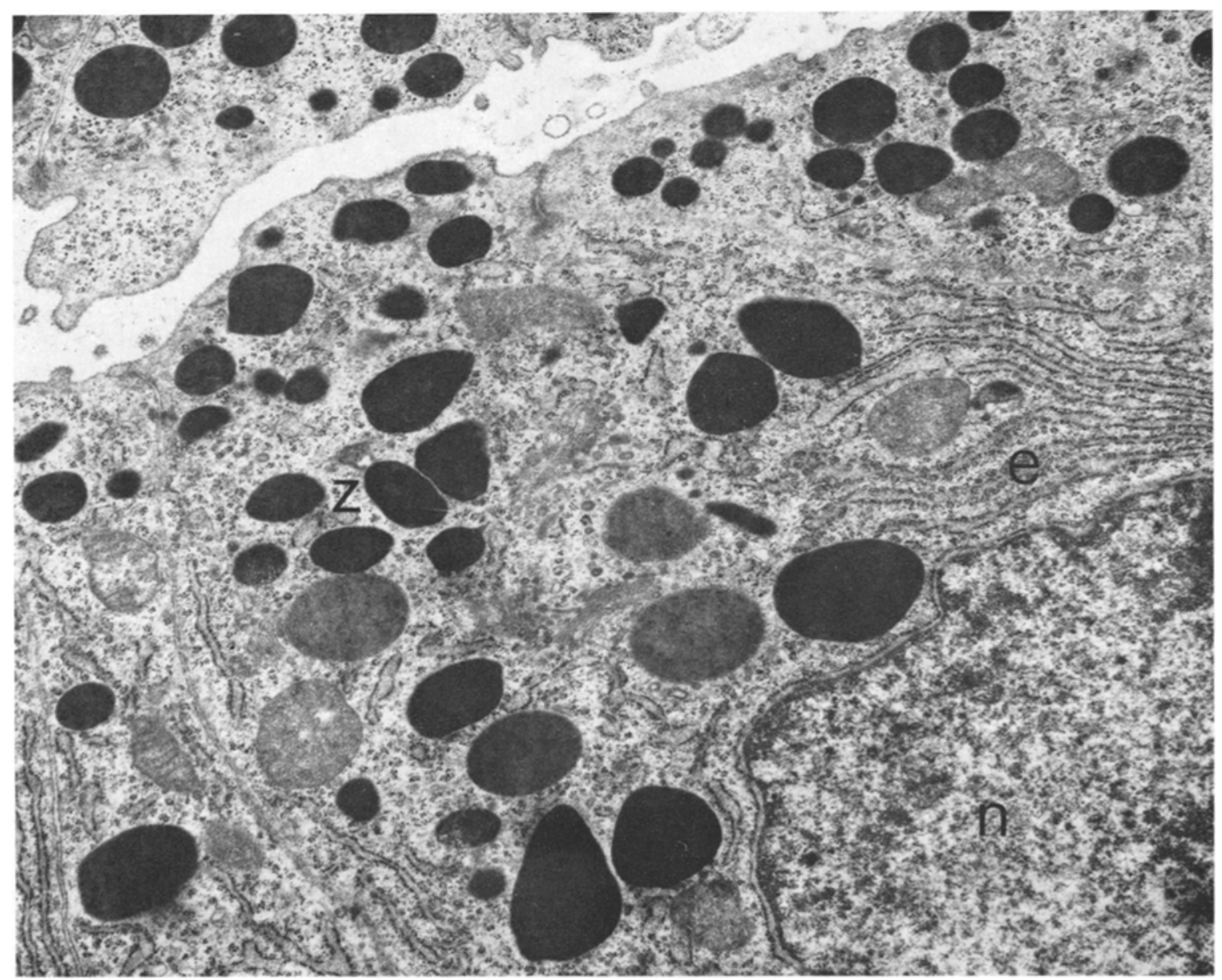

Fig. 6. Foetus de 18 jours. Cellules exocrines. Grains de zymogène $(z)$ nombreux et volumineux. $n$ : noyau. $e:$ ergastoplasme $(\times 15600)$ 
des cellules exocrines est assez variable. Les cellules renfermant des grains de zymogène sont plus riches en citernes ergastoplasmiques que les cellules morphologiquement indifférenciées mais présentent encore de très nombreux ribosomes libres. Comme les cellules morphologiquement indifférenciées, elles montrent une activité mitotique (Fig. 5).

A partir de 18 jours, les régions les plus riches en cellules endocrines peuvent être repérées sur coupes semi-fines au microscope à contraste de phase. Elles apparaissent plus pâles, dépourvues de grains de
Les cellules exocrines s'enrichissent en grains de zymogène. Ceux-ci sont nettement plus nombreux et plus volumineux qu'au stade précédent (leur plus grand diamètre atteint $1500 \mathrm{~nm}$ ). En même temps que se forment les grains de zymogène, l'ergastoplasme se développe progressivement sous forme de longues citernes à contenu dense qui tendent à s'organiser parallèlement (Fig. 6). Ces cellules morphologiquement différenciées présentent encore une activité mitotique.

A 19 jours, la taille des îlots endocrines augmente et les cellules A tendent à se disposer préférentiellement

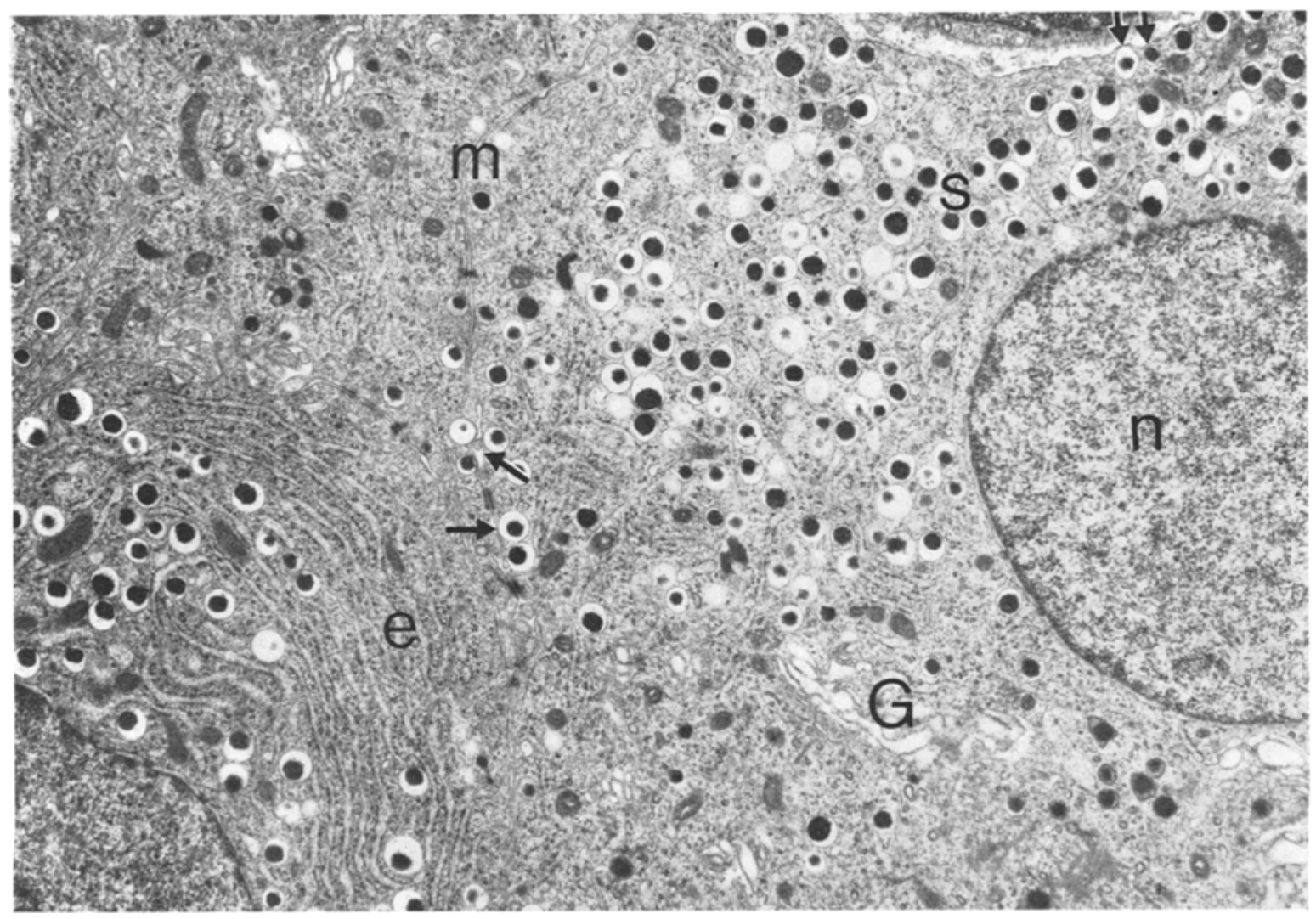

Fig. 7. Foetus de 20 jours. Cellules B. Grande richesse en matériel sécrétoire $(s)$, certains granules étant accolés (flèches) à la membrane plasmique $(m) . e$ : ergastoplasme abondant. $g$ : golgi développé. $n:$ noyau $(\times 8400)$

zymogène, ces derniers étant déjà abondants dans le tissu exocrine.

A 18 jours, les cellules endocrines morphologiquement différenciées sont groupées en petits îlots renfermant soit une seule catégorie cellulaire, $A$ ou $B$, soit un mélange des deux types de cellules. Quoique typiquement endocrines, ces cellules entourent souvent des canalicules dont elles peuvent border directement la lumière, sans interposition de cellule canalaire; dans ce cas il arrive qu'elles soient au voisinage immédiat des cellules exocrines morphologiquement différenciées, le long des mêmes canalicules. Elles présentent les mêmes caractères morphologiques qu'au stade précédent. à la périphérie (cette organisation de type adulte est plus nette le jour suivant). Du point de vue cytologique, un certain nombre de cellules endocrines présentent des signes de maturation plus marqués et sont comparables par le développement de l'ergastoplasme, de l'appareil de Golgi et le nombre des granules sécrétoires typiques aux cellules du jeune rat.

A 20 jours, les îlots, par la disposition des cellules et leur richesse en matériel sécrétoire, évoquent ceux du jeune rat; ce stade est caractérisé par la présence de nombreuses cellules morphologiquement bien différenciées dans des îlots maintenant volumineux (Figs. 7, $8,9,10$ et 11 ). La maturation des cellules $B$ se traduit par le développement considérable des structures 
ergastoplasmiques et golgiennes et par une accumulation importante de granules sécrétoires dont la taille a augmenté (à 17 et 18 jours les plus gros mesuraient $180 \mathrm{~nm}$ et à 20 jours ils atteignent $430 \mathrm{~nm}$ ). Les complexes golgiens présentent un aspect caractéristique d'amas compacts de saccules clairs plus ou moins dilatés. Les vésicules contenant les granules sécrétoires
Nous avons rencontré quelques cellules B munies d'un cil. Ces formations ciliaires n'ont rien de spécifique et nous ne les signalons ici qu'à titre de curiosité. Nous les avons fréquemment observées dans les cellules indifférenciées bordant les canalicules. Leur présence dans les cellules endocrines du pancréas est d'ailleurs classique $[19,25]$.

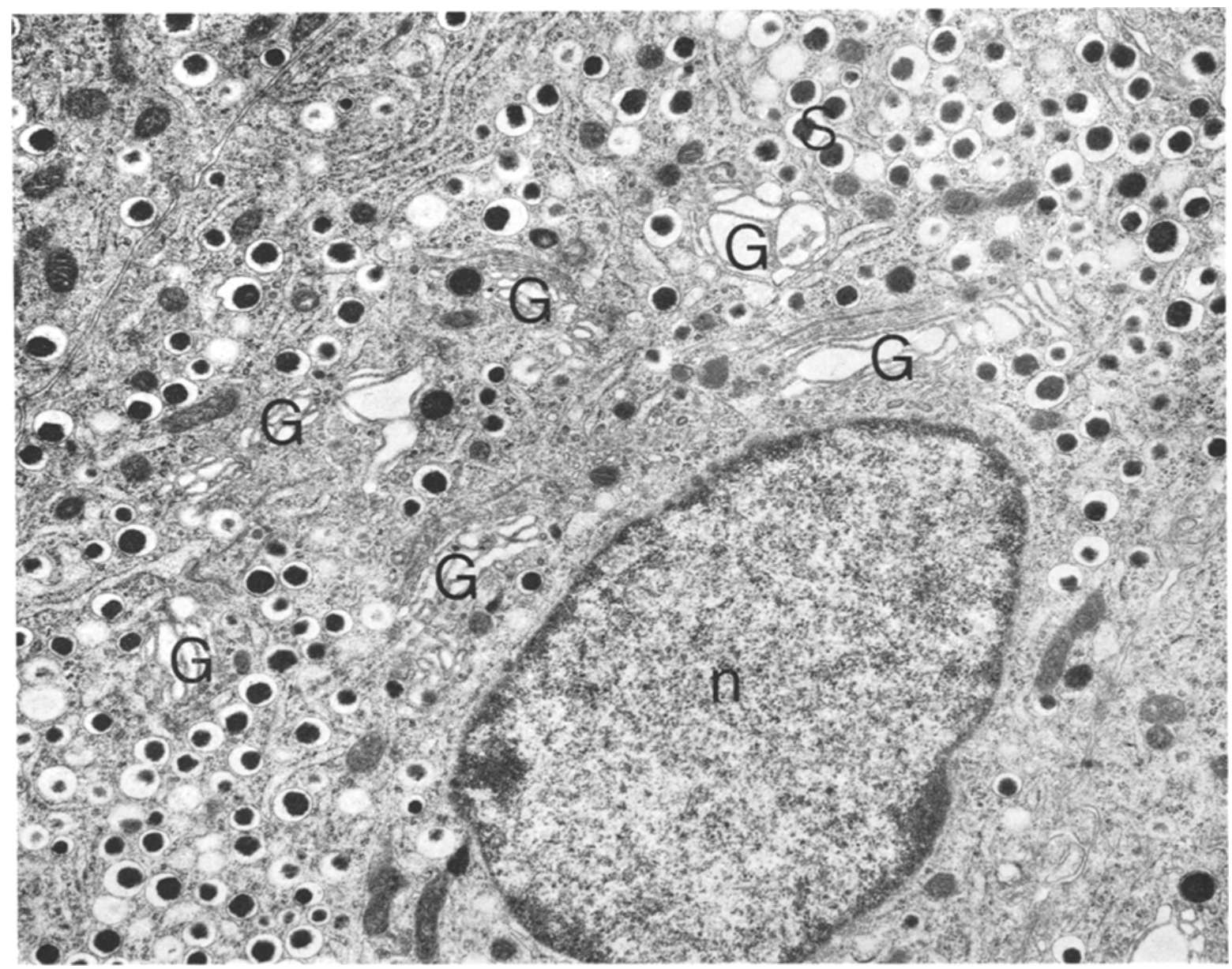

Fig. 8. Foetus de 20 jours. Cellule B montrant un golgi $(g)$ très développé. $(s)$ : grains de sécrétion. $n$ : noyau $(\times 11600)$

à tendance cristalloïde sont également dilatées (Figs. 7 et 8 ).

La maturation des cellules A se traduit surtout par l'augmentation très nette du nombre et de la taille des granules sécrétoires (la taille des plus gros granules est de $180 \mathrm{~nm}$ à 17 et 18 jours et $300 \mathrm{~nm}$ à 20 jours); ergastoplasme et golgi ne présentent pas de modifications appréciables (Fig. 9).

Dans les deux types cellulaires, on trouve dès les premiers stades de différenciation morphologique des granules accolés à la membrane plasmique, aussi bien dans les régions latérales qu'au pôle basal de la cellule (Figs. 7 et 10 par exemple). Des cellules endocrines morphologiquement différenciées sont vues en mitose.
On ne note pas de variation appréciable des îlots entre 20 et 21 jours.

Vers la fin de la gestation, des filets nerveux peuvent être observés au voisinage immédiat des cellules endocrines, à la périphérie des îlots (Fig. 12).

L'évolution du pancréas exocrine entre 19 jours et la naissance se traduit par une discrète augmentation de la taille des grains de zymogène, la richesse des cellules en ergastoplasme reste inférieure à celle observée chez le jeune rat.

\section{Discussion}

Jusqu'à 18 jours, les cellules endocrines rencontrées dans le pancréas foetal du rat conservent, par le nombre 
relativement restreint des granules sécrétoires et la richesse en ribosomes libres, un aspect relativement immature (Figs. 2, 3 et 4). A partir de 19 jours au contraire une maturation est visible: l'organisation des îlots tend vers un type adulte et entre 19 et 20 jours les caractères morphologiques des cellules endocrines évoluent rapidement vers ceux que l'on rencontre chez le jeune de 8 jours (Figs. 7, 8, 9, 10 et 11). Cette maturation correspond à l'augmentation très nette de gique du tissu exocrine observée ici correspond vraisemblablement à cette dernière phase d'accroissement rapide. Ceci implique qu'avant ce stade les cellules exocrines ont subi leur différenciation physiologique sans qu'une différenciation morphologique soit évidente. Ceci est en accord avec les travaux de Kulka [14] sur le pancréas exocrine de poulet. Il est difficile de savoir si semblable remarque peut s'appliquer aux cellules endocrines.

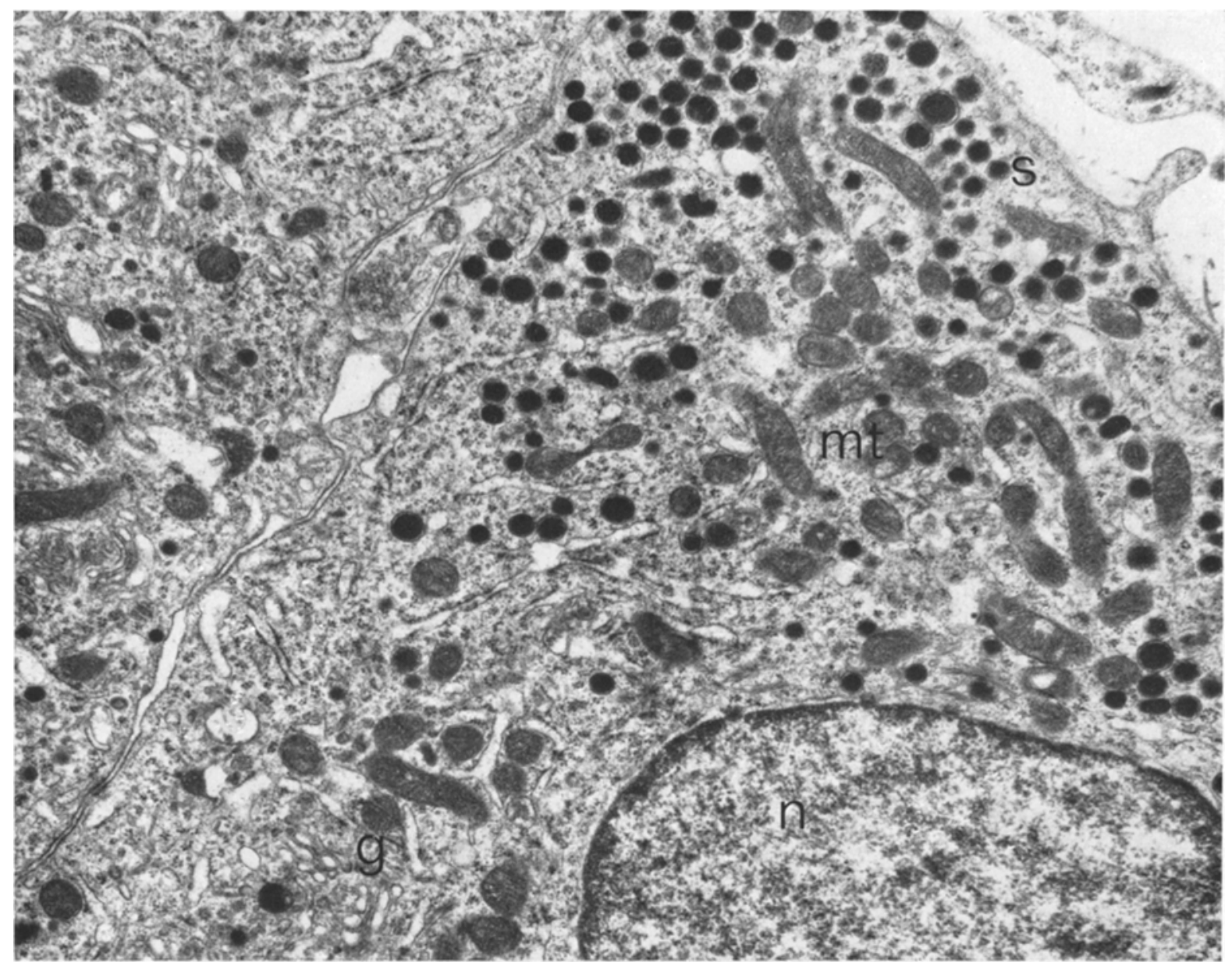

Fig. 9. Foetus de 20 jours. Cellules A. Granules sécrétoires $(s)$ nombreux, golgi $(g)$ discret. $m t$ : mitochondries. $n: \operatorname{noyau}(\times 15600)$

l'insulinémie foetale à 20 jours [3], à l'accroissement de la concentration pancréatique en insuline $[2,18,24]$ et au maximum de concentration pancréatique en glucagon observé à 20 jours $[20,18]$.

Les cellules exocrines morphologiquement différenciées deviennent nombreuses à 17 jours mais ce n'est qu'à partir de 18 jours que la richesse en grains de zymogène devient appréciable (Figs. 5, 6). Ensuite de 19 jours à la naissance l'évolution est discrète. Des dosages ont révélés la présence d'enzymes digestifs dans le pancréas à partir de 12 jours de gestation et le brusque accroissement de la teneur en ces enzymes entre 16 et 19 jours [27]. La différenciation morpholo-

\section{Bibliographie}

1. Caramia, F.: Electron microscopic description of a third cell type in the islets of the rat pancreas. Amer. J. Anat. 112, 53-64 (1963).

2. Dixit, P. K., Lowe, I. P., Heggestad, C. B., Lazarow, A.: Insulin content of microdissected fetal islets obtained from diabetic and normal rats. Diabetes 13, 71-77 (1964).

3. Félix, J.M., Jacquot, R., Sutter, B.Ch.J.: Insulinémies maternelles et foetales chez le rat. Horm. and Metab. Res. 1, 41-42 (1969).

4. Ferreira, D.: L'ultrastructure des cellules du pancréas endocrine chez l'embryon et le rat nouveau-né. J. Ultrast. Res. 1, 14-25 (1957). 


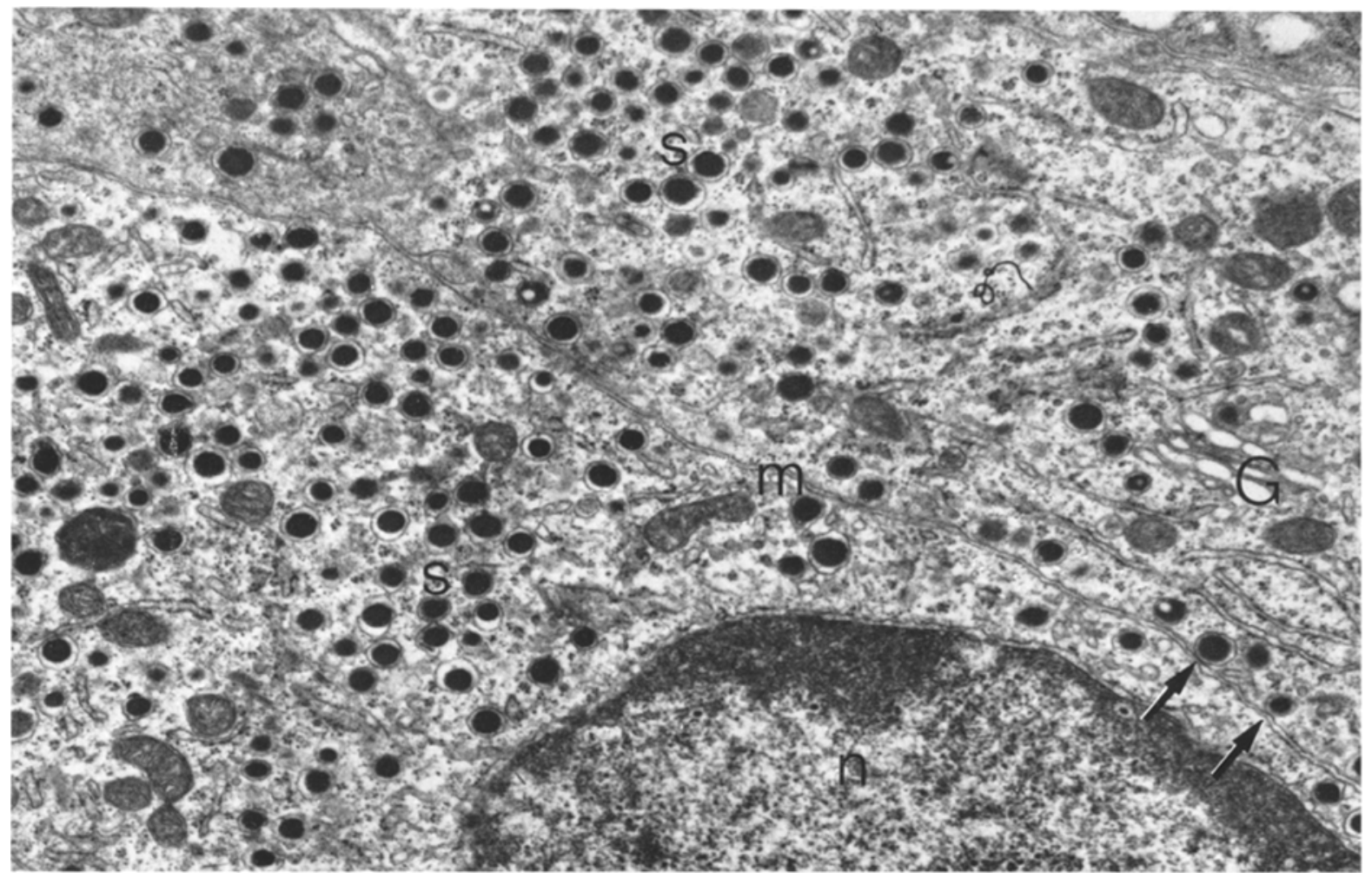

Fig. 10. Jeune de 8 jours. Cellules A. Granules secrétoires (s) nombreux et parfois accolés (flèches) à la membrane plasmique $(m)$. Golgi $(g)$ discret. $n$ : noyau. ( $\times 11600$ )

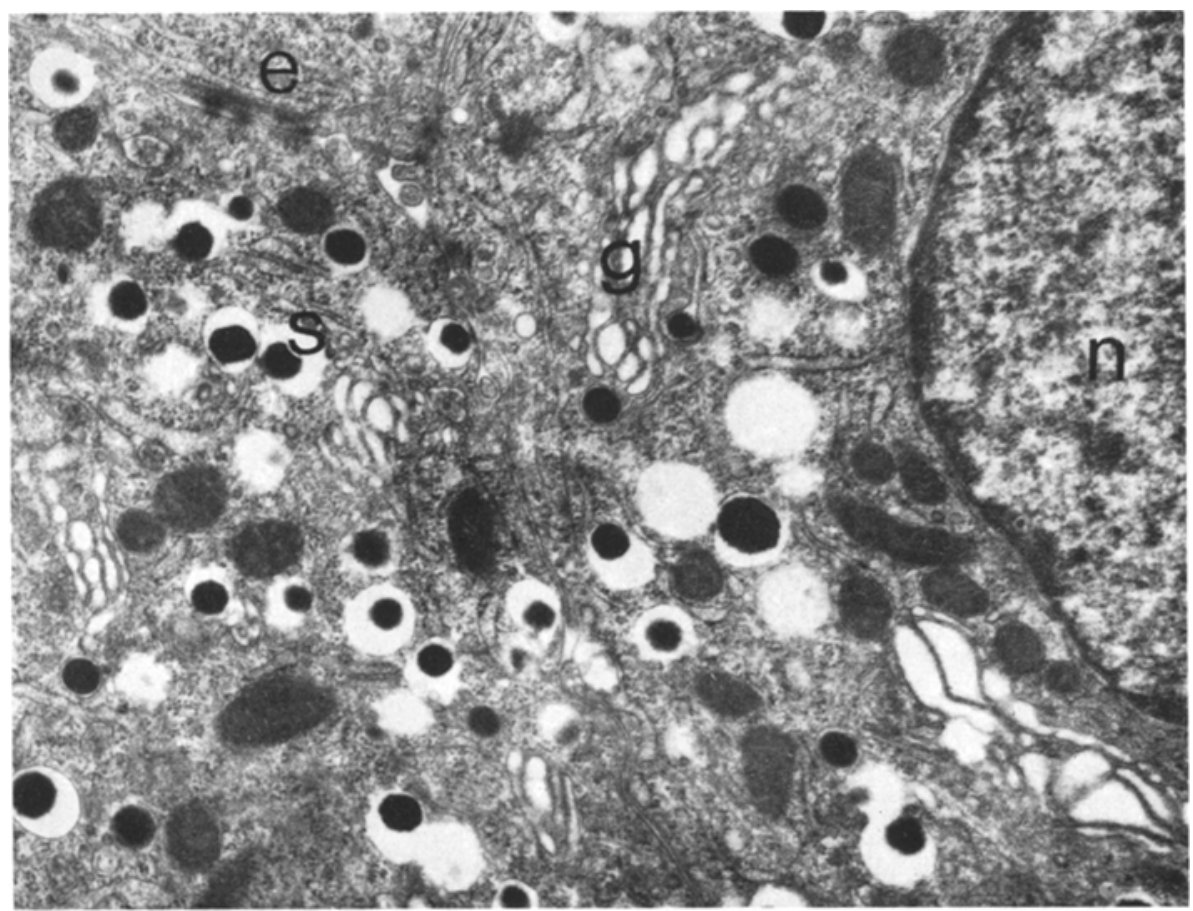

Fig. 11. Jeune de 8 jours. Cellules B matériel sécrétoire $(s)$ important, structures ergastoplasmiques $(e)$ et golgiennes $(g)$ bien développées. $n:$ noyau ( $\times 15600$ ) 


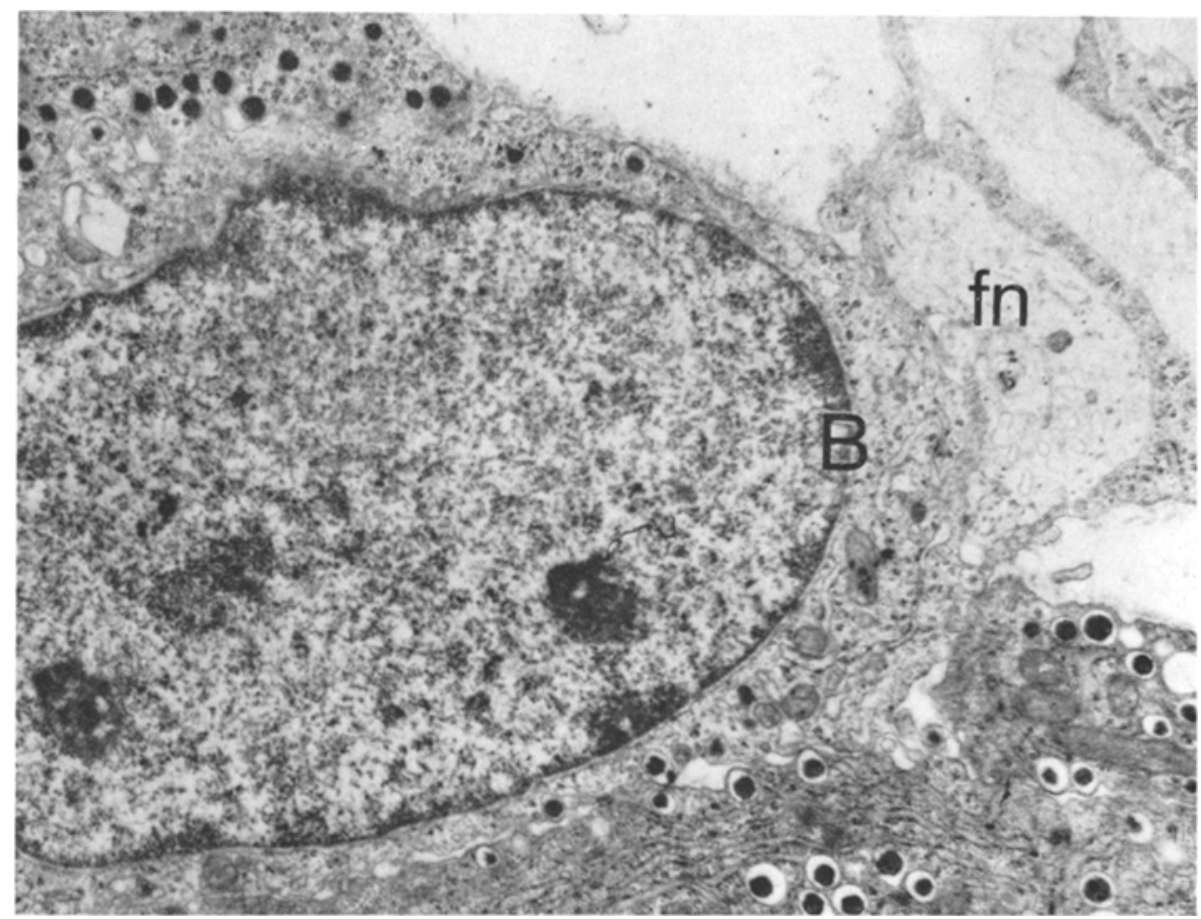

Fig. 12. Foetus de 21 jours. Filet nerveux (f. n.) au voisinage immédiat d'une cellule B (B) $(\times 11600)$

5. Gonet, A.E.: Studies on transplantation of fetal rat pancreas. In: The symposium on structure and metabolism of the pancreatic islets, p. $179-187$. Eds.: Brolin, S.E., Hellman, B., Knutson, H. Oxford: Pergamon Press 1964.

6. Greengard, O., Dewey, H.K.: Initiation by glucagon of the premature development of tyrosine aminotransferase, serine dehydratase, and glucose-6-phosphatase, in fetal rat liver, J. biol. Chem. 2986-2991 (1967).

7. Grillo, T.A.I. The occurence of insulin in the pancreas of foetuses of some rodents. J. Endocr. 31, 67-73 (1964).

8. Hard, W.L.: The origin and differentiation of the alpha and beta cells in the pancreatic islets of the rat. Amer. J. Anat. 75, 369-403 (1944).

9. Heggestad, C., Morgan, C.R., Lazarow, A.: Insulin levels in maternal and fetal plasma from normal and alloxan diabetic rats near term. Anat. Rec. 151, 455$456(1965)$.

10. Hellerström, C., Hellman, B.: Reactions of the two types of A cells in the islets of Langerhans after administration of glucagon. Acta endocr. 41, 116-122 (1962).

11. - - Petersson, B., Alm, G.: The two types of pancreatic $A$ cells and their relation to the glucagon secretion. In: The symposium on the strueture and metabolism of pancreatic islets, 117-130. Eds.: Brolin, S.E., Hellman, B., Knutson, H. Oxford: Pergamon Press 1964.

12. Hellman, B.: The development of the mammalian endocrine pancreas. Biol. Neonat. 9, 263-278 $(1965 / 66)$.

13. Jacquot, R.: Some hormonally controlled events of liver differentiation in the perinatal period. In, Proceedings of the conference on "Hormones in Development" Nottingham, the National Foundation, September 1968 (sous presse).
14. Kulka, R. G., Yaloysky, U.: Secretion of $\alpha$-amylase by the embryonic chick pancreas in vitro. $J$. cell. Biol, 29, 287-292 (1966).

15. Lacy, P.E.: Electron microscopic identification of different cell types in the islets of Langerhans of the guinea pig, rat, rabbit and dog. Anat. Ree. 128, 255267 (1957).

16. Lambert, A.E., Junod, A., Stauffacher, W., Jeanrenaud, B., Renold, A. E.: Organ culture of fetal rat pancreas. I. Insulin release induced by caffeine and by sugars and some derivatives. Biochem. biophys. Acta 184, 529-539 (1969).

17. - Jeanrenaud, B., Junod, A., Renold, A. E.: Organ culture of fetal rat pancreas. II. Insulin release induced by amino and organic acids, by hormonal peptides, by cationic alterations of the medium and by other agents. Biochem. biophys. Acta 184, 540-553 (1969).

18. - Orci, L., Junod, A. F., Jeanrenaud, B., Renold, A. E., Rouiller, Ch.: Biological and morphological studies on fetal and newborn rat pancreas. Fourth annual meeting of the European Association for the Study of Diabetes-Abstracts, in Diabetologia 5, 46 (1969).

19. Munger, B.L.: A light and electron microscopic study of cellular differentiation in the pancreatic islets of the mouse. Amer. J. Anat. 103, 275-312 (1958).

20. Orci, L., Lambert, A. E., Rouiller, Ch., Renold, A.E., Samols, E.: Evidence for the presence of A-cells in the endocrine foetal pancreas of the rat. Horm. Metab. Res. 1, 108-110 (1969).

21. Perrier, H., Porte, A., Jacquot, R.: Présence de cellules $\mathrm{A}$ dans le pancréas foetal de rat. C.R. Acad. Sci. 269, $841-843(1969)$.

22. Petersson, B., Hellman, B.: Effects of long term administration of glucagon on the pancreatic islet tissue of rats and guinea-pigs. Acta endocr. 44, 139--149 (1963). 
23. Pictet, R., Clark, W.R., Renold, A.E., Williams, R. H., Rutter, W.J.: An electron microscope study of the in vitro development of the embryonic rat pancreas. J. cell. Biol. 39, 105 (1968).

24. Rishi, S., Golob, E.K., Becker, K. L., Shah, N.: Pancreatic insulin content of nonpregnant, pregnant and pospartum rats and the developing rat fetus. Diabetes 18, 268-272 (1969).

25. Theret, C., Tamboise, E.: Formations ciliaires observées au microscope électronique dans des cellules sécrétoires néoformées de l'adénohypophyse et du pancréas endocrine. C.R. Acad. Sci. 256, 2025-2027 (1963).
26. Vecchio, D., Luyckx, A., Zahnd, G.R., Renold,A.E.: Insulin release induced by glucagon in organ cultures of fetal rat pancreas. Metabolism 15, 577-581 (1966). 27. Wessells, N.K., Rutter, W.J.: Phases in cell differentiation. Scient. Amer. 220, 36-44 (1969).

Huguette Perrier

Laboratoire de Physiologie Animale et Centre de Biologie et Biochimie du Développement

Faculte des sciences B.P. $n^{\circ} 347$,

F-51 Reims 